\title{
CPIS Lung Ultrasound and the Erratic March toward Diagnostic Certainty in VAP
}

\author{
Ram E Rajagopalan®
}

Keywords: Clinical pulmonary infection score, Diagnostic imaging, Lung ultrasound, Ventilator-associated pneumonia.

Indian Journal of Critical Care Medicine (2021): 10.5005/jp-journals-10071-23751

Despite its frequent occurrence in the intensive care setting, the diagnosis of VAP is confounded by the unavailability of diagnostic tests with appropriate performance characteristics. Clinical evaluation and standard radiological examinations at the bedside lack both sensitivity and specificity. ${ }^{1}$ As a consequence, most clinicians rely on microbiological cultures of airway secretions to arrive at a diagnosis.

Unfortunately, routine qualitative cultures of suctioned tracheal secretions lack specificity for the detection of VAP, as airway colonization and tracheobronchitis may not be differentiated by such cultures. Studies that have used postmortem lung histology as the gold standard for VAP consistently report excellent specificity for dense microbial growth on quantitative cultures taken from the distal airway. ${ }^{2,3}$ Thus, high quantitative growth $\left(>10^{4} \mathrm{cfu} / \mathrm{mL}\right)$ on the BAL raises the specificity of diagnosis of pneumonia from $\sim 75 \%$ (with endotracheal aspirates) to as high as $100 \%$. $^{3}$

The process of obtaining distal bronchial secretions by BAL, however, is cumbersome and potentially decompensating in ventilated patients with severe hypoxia. Limited bronchoscopic skills, the high costs, and restricted availability of quantitative cultures impose constraints on their routine use in India. So, is a test with high specificity desirable or even necessary?

Two randomized control studies have compared outcomes in VAP diagnosed by an invasive technique (BAL-directed distal airway cultures; high specificity) with a noninvasive method of airway sampling (nonquantitative culture of tracheal aspirate; high sensitivity). Neither investigation was able to demonstrate a difference in unadjusted 28-day mortality or in ventilator-free days. ${ }^{4,5}$

While this may be cursorily interpreted as sufficient support for noninvasive diagnosis, the higher sensitivity of qualitative endotracheal aspirates and the more frequent isolation of organisms in suspected VAP result in overtreatment with antibiotics, $^{4,6,7}$ which may promote antibiotic resistance. On this basis, professional consensus in Europe, for instance, is to perform quantitative BAL before initiating antibiotics in all patients with suspected VAP.

This dilemma, where the benefits of an effective, easily available, and inexpensive process like tracheal aspirate culture are countered by concerns about the overuse of antibiotics, needs resolution in the context of settings with major antibiotic resistance issues. It is in this framework that we should reassess the value of simple clinical tools such as the CPIS that may provide us with the specificity without needing complex invasive processes.
Department of Clinical Services, Sri Ramachandra Institute of Higher Education and Research, Chennai, Tamil Nadu, India

Corresponding Author: Ram E Rajagopalan, Department of Clinical Services, Sri Ramachandra Institute of Higher Education and Research, Chennai, Tamil Nadu, India, Tamil Nadu, India, Phone: +91 9841010859, e-mail: rajagopalan.ram@gmail.com

How to cite this article: Rajagopalan RE. CPIS Lung Ultrasound and the Erratic March toward Diagnostic Certainty in VAP. Indian J Crit Care Med 2021;25(3):255-257.

Source of support: Nil

Conflict of interest: None

\section{Is the CPIS Specific Enough?}

The CPIS as developed by Pugin and colleagues was an "attempt to determine early (clinical) criteria for the diagnosis of pulmonary infection" ${ }^{8}$ against the surrogate gold standard of quantitative culture of BAL fluid. In this study, the CPIS determined by six easily assessable criteria was able to discriminate between the presence and absence of microbiologically defined pneumonia with excellent sensitivity and specificity. A CPIS cutoff score of six demonstrated $93 \%$ sensitivity and $100 \%$ specificity in identifying pneumonia.

The optimism of this initial study in utilizing a simple clinical tool to increase the specificity of our diagnosis of VAP did not withstand subsequent rigorous re-evaluation. ${ }^{9}$ While methodological issues related to the small initial study in a homogeneous group of mainly medical patients limit its extrapolation, the constituent parameters used in the score were arbitrarily selected and weighted based on expert opinion and not on their true predictive value. As a consequence, further testing against histological and quantitative microbiological standards yielded far lower specificities, ranging from 42 to $85 \% .{ }^{9}$ In a post hoc analysis of the large database from the Canadian Critical Care Trials Group's (CCTG) RCT comparing invasive and noninvasive diagnosis of $\mathrm{VAP}^{4}$ the CPIS performed poorly and was unable to discriminate between the presence and absence of VAP assessed by clinical adjudication (AU-ROC; 0.47 ; $95 \% \mathrm{Cl} ; 0.42-0.53) .^{10}$

\section{Ultrasound to the Rescue}

Some of the poor performance of the CPIS may be attributed to its irreproducibility-being highly variable when assessed by multiple clinical observers. ${ }^{9}$ The subjectivity in the interpretation 
of some of the included parameters such as the chest radiograph has been a major issue. ${ }^{1,9}$ Based on this concern, Samanta et al., the investigators of a study published in this issue of IJCCM, ${ }^{11}$ have substituted the radiographic constituents of the CPIS with sonographic findings to generate a "sono-pulmonary infection score" (SPIS) presuming greater specificity and inter-rater reliability of the ultrasound evaluation.

How robust are these sonographic parameters? After the initial description of lung ultrasound correlates of pneumonia in critically ill patients by Lichtenstein and colleagues, a series of smaller studies have compared the diagnostic value of LUS and bedside chest X-ray against the standard of chest CT. An earlier systematic review of these studies claimed similar specificity for LUS and chest $\mathrm{X}$-ray. ${ }^{12}$ However, these studies did not look at the sonographic characteristics of consolidation but focused only on their presence along with pneumothorax and pleural effusion. A more nuanced understanding of LUS features has emerged since then, ${ }^{13,14}$ and the appearance of anterior subpleural consolidations and lobar/ sublobar consolidations with a dynamic air bronchogram has been identified as being more specific (80-90\%) for VAP in prospective cohorts ${ }^{13,14}$ even using quantitative BAL as the comparator. ${ }^{13}$ The authors of the current study ${ }^{11}$ have used these parameters for inclusion in their SPIS.

\section{The Current Study}

Samanta and colleagues show that the substitution of LUS parameters in the place of chest radiography increases the discrimination of the CPIS (AU-ROC; 0.617 for CPIS increases to 0.808 for SPIS) and in the specificity of diagnosis of VAP. The rise in specificity (with a cutoff score of 6) from $60 \%$ for CPIS $(95 \% \mathrm{Cl}$; 40.6-77.3) to $73.3 \%$ for SPIS ( $95 \% \mathrm{Cl} ; 54.1-87.7)$ is clinically important and is augmented further by the inclusion of culture results.

Unfortunately, the overlapping 95\% confidence limits in this study warrant a cautious interpretation of these hopeful results and it may be worthwhile to keep the following considerations in mind:

- The use of quantitative culture of mini-BAL aspirate as the surrogate standard for VAP in this paper may be questioned. Studies imply that its performance (lower specificity) may not be equivalent to conventional invasive $B A L,{ }^{2,3}$ which would have been the ideal comparator to judge the performance of the SPIS.

- Though the prospective nature of this study assures us of the quality of the study data, the hypothesis that lung ultrasound would augment the predictive value of CPIS was, at best, conjunctural, based on its performance characteristics in other situations. The magnitude of change in specificity or the AU-ROC could not be predicted in advance to plan a study of appropriate size. As a result, the clinically important benefits in specificity seen in this study do not reach statistical significance (overlapping 95\% confidence limits) and need confirmation in a larger data set.

- The use of an expert radiologist to perform LUS may affect the extrapolatability of this SPIS study to many Indian ICUs where point-of-care sonography may be performed by the primary intensivist. The greater reliability of an evaluation by a trained radiologist in this study may exaggerate its performance. In fact, the available data on inter-rater variability imply weak to minimal agreement among nonexpert clinicians in the LUS assessment of consolidation ( $\mathrm{K}=0.39-0.57)$ and subpleural consolidation $(\mathrm{K}=0.31-0.49)^{15,16}$
- It is unfortunate that the authors seem to have dichotomized the CPIS and SPIS by the traditional CPIS cutoff score of 6 . This may be arbitrary, and an exploration of the performance of other cutoff scores, especially for the SPIS, may have identified a different specificity for this test.

While it may be tempting to immediately generalize the diagnostic methodology suggested by this elegant study to clinical practice, the exploratory nature of the SPIS cannot be overemphasized. Independent prospective validation in studies of a sufficient size will be needed to confirm the gain in specificity identified in the current investigation. It also remains to be seen, if this gain in specificity translates into more restrictive antibiotic use, as, going by prior experience, ${ }^{4,5}$ it is unlikely to influence clinical outcomes and survival. Finally, most of the components of the CPIS seem to have no independent predictive value in large prospective databases, with the exception of oxygenation (P/F ratio)..$^{17}$ It may be time to abandon the CPIS/SPIS paradigm entirely and combine the highly specific LUS findings with alternative parameters like airway secretion microscopy/culture, biomarkers, and oxygenation indices to develop better predictive tools for VAP.

The path to diagnostic certainty is often not steady or linear, but the information gathered by such exploratory evaluations is certainly pointing us in the right direction.

\section{Abbreviations}

VAP Ventilator-associated pneumonia

BAL Bronchoalveolar lavage

CPIS Clinical pulmonary infection score

AU-ROC Area under the receiver operating characteristic curve CT Computerized tomography

$\mathrm{P} / \mathrm{F} \quad \mathrm{PaO}_{2} / \mathrm{FiO}_{2}$ ratio

LUS Lung ultrasound

\section{ORCID}

Ram E Rajagopalan (i) https://orcid.org/0000-0001-9486-3285

\section{References}

1. Klompas M. Does this patient have ventilator-associated pneumonia? JAMA 2007;297(14):1583-1593. DOI: 10.1001/jama.297.14.1583.

2. Rouby JJ, De Lassale EM, Poete P, Nicolas MH, Bodin L, Jarlier V, et al. Nosocomial bronchopneumonia in the critically ill. Histologic and bacteriologic aspects. Am Rev Respir Dis 1992;146(4):1059-1066. DOI: 10.1164/ajrccm/146.4.1059.

3. Marquette CH, Copin MC, Wallet F, Neviere R, Saulnier F, Mathieu D, et al. Diagnostic tests for pneumonia in ventilated patients: prospective evaluation of diagnostic accuracy using histology as a diagnostic gold standard. Am J Respir Crit Care Med 1995;151:1878-1888. DOI: 10.1164/ajrccm.151.6.7767535.

4. Fagon JY, Chastre J, Wolff M, Gervais C, Parer-Aubas S, Stéphan F, et al. Invasive and noninvasive strategies for management of suspected ventilator-associated pneumonia-a randomized trial. Ann Intern Med 2000;132(8):621-630. DOI: 10.7326/0003-4819-132-8-20000418000004.

5. The Canadian Critical Care Trials Group. A randomized trial of diagnostic techniques for ventilator-associated pneumonia. N Engl J Med 2006;355:2619-2630. DOI: 10.1056/NEJMoa052904.

6. Shorr AF, Sherner JH, Jackson WL, Kollef MH. Invasive approaches to the diagnosis of ventilator-associated pneumonia: a meta-analysis. Crit Care Med 2005;33(1):46-53. DOI: 10.1097/01.ccm.0000149852. 32599.31. 
7. Torres A, Niederman MS, Chastre J, Ewig S, Fernandez-Vandellos $\mathrm{P}$, Hanberger $\mathrm{H}$, et al. Guidelines for the management of hospitalacquired pneumonia (HAP)/ventilator-associated pneumonia (VAP) of the European Respiratory Society (ERS), European Society of Intensive Care Medicine (ESICM), European Society of Clinical Microbiology and Infectious Diseases (ESCMID) and Asociación Latinoamericana del Tórax (ALAT). Eur Respir J 2017;50(3):1700582. DOI: 10.1183/13993003.00582-2017.

8. Pugin J, Auckenthaler R, Mili N, Janssens JP, Lew PD, Suter PM. Diagnosis of ventilator-associated pneumonia by bacteriologic analysis of bronchoscopic and nonbronchoscopic "blind" bronchoalveolar lavage fluid. Am Rev Respir Dis 1991;143(5 Pt 1):1121-1129. DOI: 10.1164/ajrccm/143.5_Pt_1.1121.

9. Zilberberg MD, Shorr AF. Ventilator-associated pneumonia: the clinical pulmonary infection score as a surrogate for diagnostics and outcome. Clin Infect Dis 2010;51(S1):S131-S135. DOI: 10.1086/ 653062.

10. Lauzier F, Ruest A, Cook D, Dodek P, Albert M, Shorr AF, et al. The value of pretest probability and modified clinical pulmonary infection score to diagnose ventilator-associated pneumonia. J Crit Care 2008;23(1):50-57. DOI: 10.1016/j.jcrc.2008.01.006.

11. Samanta S, Patnaik R, Azim A, Gurjar M, Baronia AK, Poddar B, et al. Incorporating lung ultrasound in clinical pulmonary infection score as an added tool for diagnosing ventilator-associated pneumonia: a prospective observational study from a tertiary care center. Indian $J$ Crit Care Med 2021;25(3):284-291.

12. Winkler MH, Touw HR, van de Ven PM, Twisk J, Tuinman PR. Diagnostic accuracy of chest radiograph, and when concomitantly studied lung ultrasound, in critically ill patients with respiratory symptoms: a systematic review and meta-analysis. Crit Care Med 2018;46(7):e707e714. DOI: $10.1097 / \mathrm{ccm} .0000000000003129$.

13. Mongodi S, Via G, Girard M, Rouquette I, Misset B, Braschi A, et al. Lung ultrasound for early diagnosis of ventilator-associated pneumonia. Chest 2016;149(4):969-980. DOI: 10.1016/j.chest.2015.12.012.

14. Staub LJ, Biscaro RRM, Maurici R. Accuracy and applications of lung ultrasound to diagnose ventilator-associated pneumonia: a systematic review. J Intensive Care Med 2018;33(8):447-455. DOI: $10.1177 / 0885066617737756$.

15. Gravel CA, Monuteaux MC, Levy JA, Miller AF, Vieira RL, Bachur RG. Interrater reliability of pediatric point-of-care lung ultrasound findings. Am J Emerg Med 2020;38(1):1-6. DOI: 10.1016/j.ajem.2019.01.047.

16. Kumar A, Weng Y, Graglia S, Chung S, Duanmu Y, Lalani F, et al. Interobserver agreement of lung ultrasound findings of COVID-19. J Ultrasound Med 2021. DOI: 10.1002/jum.15620.

17. Shorr AF, Cook D, Jiang X, Muscedere J, Heyland D, Canadian Critical Care Trials Group. Correlates of clinical failure in ventilator-associated pneumonia: insights from a large, randomized trial. J Crit Care 2008;23(1):64-73. DOI: 10.1016/j.jcrc.2007.11.010. 